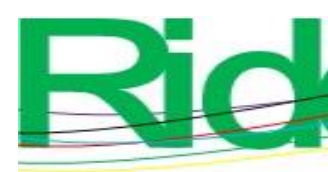

Revista Iberoamericana para la Investigación y el Desarrollo Educativo

https://doi.org/10.23913/ride.v11i22.902

Artículos científicos

\title{
La incorporación de la tutoría en una institución formadora de docentes
}

\section{The incorporation of tutoring in a teacher training school \\ Incorporação da tutoria em instituição de formação de professores}

\author{
Astrid Olimpia Martínez Hernández \\ Universidad Autónoma de Baja California, México \\ martinez.astrid@uabc.edu.mx \\ https://orcid.org/0000-0003-2445-2469 \\ Salvador Ponce Ceballos \\ Universidad Autónoma de Baja California, México \\ ponce@uabc.edu.mx \\ https://orcid.org/0000-0003-0454-9853 \\ Yaralín Aceves Villanueva \\ Universidad Autónoma de Baja California, México \\ yaralin@uabc.edu.mx \\ https://orcid.org/0000-0002-8175-2639
}

\section{Resumen}

En este artículo se presentan los resultados de un ejercicio investigativo de tipo cualitativo apoyado en el análisis de contenido que buscó diagnosticar de forma participativa la pertinencia de crear de manera formal un programa de tutorías académicas en una escuela normal en el norte de México. Los resultados dan cuenta de la importancia y existencia no formal de las tutorías, así como de la delimitación de algunas orientaciones para el diseño de un programa oficial. De igual forma, se detecta una valoración positiva por parte de los participantes sobre la importancia de la colegiación con los actores para el diseño de este tipo de estrategias. Las conclusiones apuntan a la pertinencia de la incorporación de las tutorías 


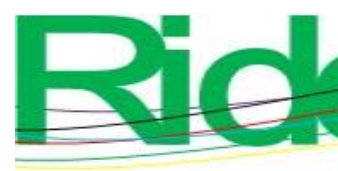
Revista Iberoamericana para la
Investigación y el Desarrollo Educativo
ISSN $2007-7467$

académicas en la escuela normal dada la trascendencia de la formación de los estudiantes y el desarrollo de los docentes.

Palabras clave: educación superior, escuela Normal, tutoría.

\begin{abstract}
The results of a qualitative research exercise supported by content analysis are presented, which sought to diagnose in a participatory way the relevance of formally creating a tutoring program in a teacher training school in Northern Mexico. The results show the importance and non-formal existence of the tutorials, as well as the delimitation of some guidelines for the design of an official program. In the same way, a positive assessment is detected by the participants on the importance of collegiality with the actors for the development of this type of strategies. The conclusions point to the relevance of the incorporation of tutoring in the teacher training school given the importance of the training of students and the development of teachers.
\end{abstract}

Keywords: Higher education, Teacher training school, Tutoring.

\title{
Resumo
}

Este artigo apresenta os resultados de um exercício de pesquisa qualitativa apoiado na análise de conteúdo que buscou diagnosticar de forma participativa a relevância da criação formal de um programa de tutoria acadêmica em uma escola normal do norte do México. Os resultados mostram a importância e a existência não formal de tutoriais, bem como a delimitação de algumas orientações para a concepção de um programa oficial. Da mesma forma, é detectada uma avaliação positiva pelos participantes sobre a importância da associação com os atores para o desenho deste tipo de estratégias. As conclusões apontam para a relevância da incorporação dos tutoriais académicos na escola normal dada a importância da formação dos alunos e do desenvolvimento dos professores.

Palavras-chave: ensino superior, escola normal, tutoria.

Fecha Recepción: Mayo 2020

Fecha Aceptación: Marzo 2021 


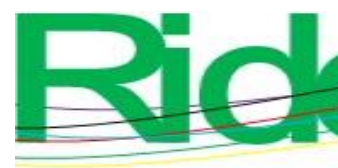

Revista Iberoamericana para la Investigación y el Desarrollo Educativo ISSN 2007-7467

\section{Introducción}

En el presente documento se describe la etapa uno de un proyecto de investigación diseñado con el objetivo de crear un programa de tutorías académicas para una escuela normal $^{1}$ en el norte de México. El proyecto se estructuró en tres etapas basadas en la metodología de Castillo y Cabrerizo (2011). En la fase inicial, se aplicó el diagnóstico; en la segunda fase, denominada ejecución, se diseñó e implementó la propuesta de un programa de tutorías, y, en la última etapa, llamada valoración, se evaluaron los resultados de la propuesta.

En este artículo, se presenta el desarrollo de la etapa uno (diagnóstico), el cual buscó integrar las opiniones de tres grupos de usuarios de una escuela normal (estudiantes, docentes y directivos) sobre la importancia de la instrumentación, en su plantel, de un programa de tutorías.

La justificación principal del desarrollo del ejercicio investigativo fue que en la institución de estudio no se contaba con un programa de tutorías, ante lo cual surgieron algunas interrogantes que fueron guiando el trabajo:

1. ¿Es importante la tutoría para los estudiantes, profesores y directivos de la institución?

2. ¿Existen antecedentes de apoyo tutorial a los estudiantes de la institución?

3. ¿Cómo debería de ser un programa de tutorías desde la óptica de sus actores: estudiantes, profesores y directivos?

En la primera parte del artículo, se presenta un marco de referencia y contextual sobre el tema de tutorías académicas. Después, se describe la metodología y, finalmente, los resultados que aportan al desarrollo de las conclusiones.

\section{Antecedentes de la tutoría académica}

La tutoría, como acompañamiento de los estudiantes, tiene sus inicios formalmente en las universidades anglosajonas (en Inglaterra, se denomina tutoring o supervising; en Estados Unidos, fue llamada mentoring, advising), donde se buscaba una educación individualizada que redujera las horas de labor del docente frente a grupo (Fresán y Romo, 2011).

\footnotetext{
${ }^{1}$ Las escuelas normales en México son un tipo de Instituciones de Educación Superior dedicadas a la formación de profesores.
} 


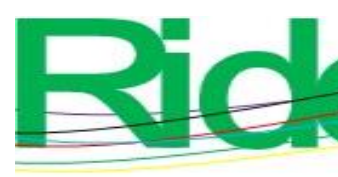

Revista Iberoamericana para la Investigación y el Desarrollo Educativo ISSN 2007 - 7467

En Canadá, Estados Unidos y algunos países de Europa, desde los años treinta, los centros de orientación son los encargados de tener una relación con el docente para orientarlo en las actividades de asesoramiento, poniendo especial atención en las necesidades académicas, sociales y personales del estudiantado (Fresán y Romo, 2011).

En Reino Unido —en la Open University, que inició en 1971—, se creó un modelo individualizado de tutorías. Al ser una universidad a distancia, los alumnos estudian autónomamente. Por ello, su modelo plantea que, en ciertos momentos, se reúnan con sus tutores, los cuales los orientan para sus siguientes etapas y les ayudan a resolver dudas que han surgido entre los estudiantes (Fresán y Romo, 2011).

En España, con la Ley de Ordenación General del Sistema Educativo (LOGSE), basándose en el Libro Blanco para la Reforma del Sistema Educativo, se señala que la tutoría es parte fundamental de la función del docente, como orientación para el alumno. Se menciona que la función del tutor es formar una relación personalizada con el estudiante para contribuir en su vida académica y para que logren su autonomía, así como su desarrollo de capacidades y actitudes para la toma de decisiones en su educación y vida social (Gobierno de España, 1989).

En Argentina, en 1997, en la Universidad Nacional del Rosario, a través de los coordinadores, se despejaban las dudas que les surgían a los nuevos estudiantes ante diversas situaciones, como trámites o cuestiones generales sobre el ámbito escolar (Torres y Luchéis, 2000, citado por Serna, 2010).

En México, alrededor de los años cuarenta, ya se experimentaban los primeros contactos con un sistema de tutorías y con la imagen de un tutor. En la Universidad Nacional Autónoma de México (UNAM), en las Facultades de Química y Ciencias Políticas y Sociales, en 1970, se definió al tutor como guía del alumno y se formalizó en los planes de estudio, lo que se convirtió en los primeros indicios de la acción tutorial (Fresán y Romo, 2011).

A partir del siglo XXI, en México se ha percibido la manifestación de las tutorías académicas dentro de las Instituciones de Educación Superior (IES). Todo esto derivado de la propuesta de los Programas Institucionales de Tutorías (PIT), planteada por la Asociación Nacional de Universidades e Instituciones de Educación Superior (ANUIES) (Fresán y Romo, 2011). 


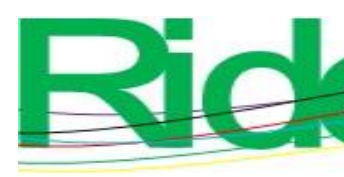

Revista Iberoamericana para la

Investigación y el Desarrollo Educativo

ISSN $2007-7467$

\section{La tutoría como actor en el trayecto educativo del estudiante}

La tutoría es una labor compleja que implica diversos ámbitos para que se ejerza de una manera pertinente y eficaz (García et al., 2016). Por lo tanto, es necesario analizar dichos ámbitos que aportan significado a la labor de la tutoría, los cuales pueden incluir la acción tutorial, los objetivos de la tutoría, el perfil del tutor, las competencias y características del tutor y las funciones del tutor, entre otros.

Según Pantoja (2013), la acción tutorial es aquella que favorece la caracterización de la educación y da la importancia de conocer individualmente las características de los estudiantes, brindándoles un apoyo académico que se adapte a sus habilidades, fortalezas y motivaciones para así ofrecerles una apropiada asesoría que vaya enfocada al ámbito educativo y profesional de los jóvenes.

En el mismo sentido, para Quintanal y García (2012) la acción tutorial es un trabajo que se realiza en conjunto con otros compañeros y con una serie de estrategias, por lo que debe ser asumido como una tarea cooperativa; además, los referidos autores destacan que la acción tutorial debe ser comparativa, lo cual se refiere a que todos, de manera coordinada, deben tener compromisos en las acciones y consecuencias que se tienen a partir de la organización acordada. Por último, mencionan que es una acción dinamizadora, donde se fortalece esa relación educativa, ya que ahí inician las tareas que se deben implementar para las condiciones concretas que se presentan en los estudiantes.

Por otra parte, García, Trejo, Flores y Rabadán (2010) titulan la acción tutorial como tutorizar, y la definen como el ejercicio de la tutoría, en el apoyo de guiar y comunicar para la formación académica y profesional de sus tutorados, de una forma personalizada o grupal. Dicha acción se debe concretar en colaboración con la propia institución y el personal que esté implicado en la labor tutorial o académica del estudiante.

\section{La tutoría académica en el contexto de escuelas normales}

La tutoría en las escuelas normales tiene sus orígenes en 2009, cuando estas fueron consideradas como Instituciones de Educación Euperior (IES) en el Programa de Mejoramiento del Profesorado (Promep), hoy Programa para el Desarrollo Profesional Docente para el Tipo Superior (Prodep), a partir del cual los docentes de tiempo completo tuvieron la responsabilidad de realizar también actividades de gestión, investigación y tutorías (Dirección General de Educación Superior Universitaria [DGESU], s. f.). 


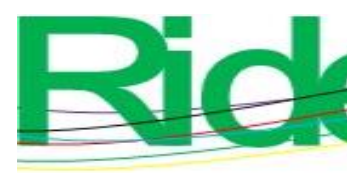

Revista Iberoamericana para la Investigación y el Desarrollo Educativo ISSN $2007-7467$

En los últimos años, una gran parte de las IES han establecido las tutorías como una estrategia para apoyar a los estudiantes durante su proceso formativo, con la finalidad de reducir el rezago y la deserción escolar, así como aumentar la eficiencia terminal.

Para lograrlo, es importante que las escuelas normales implementen y den a conocer la acción tutorial dentro de sus planteles, puesto que también pertenecen al nivel superior. Por ende, tienen la responsabilidad de asignar un tutor a sus estudiantes para lograr una adecuada trayectoria escolar y obtener un mayor número de formadores egresados y titulados.

De acuerdo con lo establecido en los planes y programas de estudios de las escuelas normales, la tutoría es el acompañamiento que se le proporciona al estudiante como un apoyo a su proceso formativo, proporcionándole las herramientas necesarias para incrementar sus experiencias académicas y orientarlo a solucionar problemáticas que se le presenten. En consecuencia, esta actividad de acompañamiento se lleva a cabo por personal docente capacitado que brinda el servicio individualmente y/o en grupos reducidos (Diario Oficial de la Federación, 2018).

En este sentido, la tutoría se entiende como el proceso de acompañamiento de los estudiantes a lo largo de su trayecto formativo, con la finalidad de guiarlo a encontrar sus habilidades y fortalezas, y que estas las utilice para resolver problemáticas que se le presenten en su vida. El porpósito consiste en volverlo autónomo para que fomente su propio aprendizaje y crecimiento personal y profesional, con lo cual aumentan las probabilidades de que consiga una estancia educativa satisfactoria y culmine sus estudios. En palabras de Tejada (2016), la tutoría en las escuelas normales tiene la siguiente finalidad:

Orientar y acompañar a los alumnos durante su proceso de formación ya sea de manera individual, que dentro de la escuela normal se realiza en pocas ocasiones, o grupal, que es lo más común en la escuela normal, para estimular mediante acciones complementarias sus potenciales, a partir del conocimiento de sus necesidades académicas, inquietudes y aspiraciones profesionales ( $\mathrm{p}$. $885)$.

En este tenor, la tutoría grupal en las escuelas normales se relaciona con la formación de los alumnos que serán futuros docentes. Por lo tanto, se aprovechan las cualidades de estos grupos para que se enriquezca el trabajo de la tutoría grupal. Tejada (2016) define la tutoría grupal académica de este modo: 


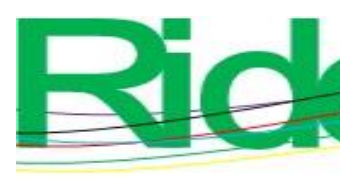

Revista Iberoamericana para la Investigación y el Desarrollo Educativo ISSN $2007-7467$

Permite rescatar la esencia de formar docentes, cuya característica principal se asocia a los procesos didácticos y pedagógicos que subyacen de esta formación. Se tiene la oportunidad de favorecer el trabajo y el aprendizaje, lo que permite el desempeño individual y colectivo de los miembros, así como observar sus competencias del proceso de formación. Además, es el espacio donde se planean dudas para su análisis y resolución en colectivo, aspecto que favorece a la reflexión de la toma de decisiones e impulsa la formación y socialización de los alumnos mediante el trabajo en equipo (p. 886).

\section{Estudios de tutorías académicas en las escuelas normales}

Existe una cantidad y variedad importante de estudios respecto a la acción tutorial en las IES. Sin embargo, en las escuelas normales, tales indagaciones aún son incipientes debido, principalmente, a la reciente incorporación formal de esa estrategia en estas últimas instituciones.

Aun así, se puede mencionar una investigación realizada en el Centro Regional de Educación Normal de Aguascalientes, la cual tuvo el objetivo de analizar el estado académico de los estudiantes con el fin de conocer las áreas de oportunidad del tutor. Los datos se obtuvieron por medio de una entrevista que expresó la vida estudiantil del participante. El estudio arrojó que hay diversos tipos de tutoría y que - con el buen uso de cada variante, según la situación que se presente — se obtienen resultados satisfactorios en los estudiantes al entrelazar las intervenciones del tutor con las acciones del Plan Institucional de Tutorías (PIT) y lo establecido en los planes y programas de estudio de la Reforma de las Escuelas Normales (Ramírez, Campos y Rodríguez, 2013).

En esta misma línea, Mapén, Pérez y López (2015) realizaron un estudio de tipo descriptivo, con un enfoque mixto, a 60 alumnos de nuevo ingreso. Aplicaron dos instrumentos: una encuesta de 18 ítems con una escala de tipo Likert y una entrevista estructurada con dos preguntas abiertas con el propósito de identificar los aspectos que ayudan a mejorar el PIT. Los resultados permitieron distinguir elementos según la perspectiva de los jóvenes normalistas, como son los horarios de atención, efectividad de la tutoría y que esta sea más personalizada. 


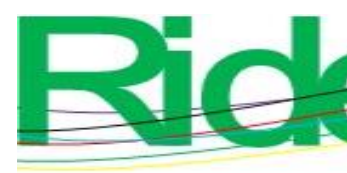

Revista Iberoamericana para la
Investigación y el Desarrollo Educativo
ISSN $2007-7467$

De forma parecida, en la Escuela Rural Lic. Benito Juárez, del municipio de Panotla, Tlaxcala, se realizó una indagación del PIT en búsqueda de mejoras. Mino, Castellanos y Gómez (2016) refieren que es de suma importancia conocer las áreas de oportunidad para una buena aplicación del PIT, ya que la mayoría de los estudiantes de su institución son de escasos recursos y la escuela cuenta con la modalidad de internado. Los autores mencionan que, debido a los grandes cambios que están viviendo las escuelas normales, la incorporación de las tutorías es esencial para lograr los cometidos.

Por otro lado, Sariñana y Sariñana (2016) realizaron una investigación de corte interpretativo en la Escuela Normal J. Guadalupe Aguilera, donde aplicaron, para la recolección de datos, entrevistas y encuestas dirigidas a estudiantes y tutores. Se enfocó en la tutoría subgrupal con el fin de desarrollar el trabajo colaborativo y la riqueza de actividades y estrategias que en ella se producen. Concluyeron que este tipo de tutoría es una estrategia pertinente para fortalecer el desarrollo integral del tutorado, aunque no todos los profesores disponen del tiempo para realizarla.

Ahumada y Obregón (2015) efectuaron una investigación un poco diferente a las anteriores en San Luis Potosí, en la Benemérita y Centenaria Escuela Normal, sin perder de vista el proporcionar una tutoría de calidad. Los participantes fueron estudiantes de la licenciatura en Educación Primaria y se utilizó el método cualitativo con alcance descriptivo. Sus técnicas de recolección de datos fueron una ficha de identificación y entrevista, cuyo cuestionario se conformó por 40 ítems para la evaluación de la autoestima. Los resultados arrojaron que los jóvenes con menor autoestima son más propensos a reprobar o desertar de la escuela, por lo que el tutor debe poner mayor importancia en los tutorados con esas características.

En la escuela normal Instituto Jaime Torres Bodet se realizó una investigación con el propósito de identificar el desempeño del docente-tutor y evaluar los beneficios y obstáculos del proceso de la tutoría, tomando en cuenta que se están formando futuros docentes. Mediante un método mixto, se utilizaron técnicas como la encuesta y un focus group con los tutores y tutorados. Se obtuvo como resultado que el obstáculo principal para llevar a cabo una tutoría académica eficiente es la falta de tiempo debido a la sobrecarga académica, así como la carencia de recursos económicos y humanos (Tejada, 2016). 


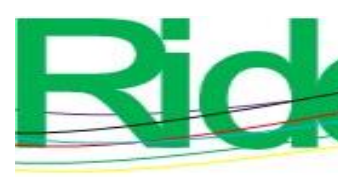

Revista Iberoamericana para la Investigación y el Desarrollo Educativo ISSN $2007-7467$

Soto, Tequida y Chávez (2017) desarrollaron una investigación de corte cualitativo en la Escuela Normal del Estado de Sonora. Utilizaron la técnica de grupos focales por medio de una entrevista semiestructurada a estudiantes de la licenciatura de Educación Preescolar. El propósito consistió en conocer su percepción sobre su labor como futuros docentes y la función de tutor que deben realizar. Los resultados indican que los jóvenes tienen conocimientos de lo que es la función tutorial, ya que expresan que es parte de su formación ser guías de sus alumnos para el desarrollo integral de ellos, por lo que es una gran responsabilidad como profesores; hacen hincapié en que esta responsabilidad es importante desarrollarla colaborativamente para recibir críticas constructivas para su mejora.

Martínez, Cortés y Contreras (2017), de la Benemérita Escuela Normal Veracruzana, se enfocaron en analizar cómo se lleva a cabo la tutoría y el impacto que esta tiene sobre las competencias que los alumnos deben desarrollar como próximos profesionales de la educación. Esta investigación se efectuó a través de una metodología de corte cualitativo, tipo etnográfico, mediante dos entrevistas semiestructuradas: una dirigida a los profesores y otra a los alumnos. Los principales hallazgos señalan que se requiere implementar un instrumento institucional con determinados parámetros, lineamientos y alcances de la función de la tutoría como herramienta de apoyo, para lograr que los alumnos desarrollen las competencias ideales a la hora de insertarse en el ámbito profesional.

Asimismo, en Mérida, Yucatán, se realizó una investigación en las tres escuelas normales estatales que operan en esa ciudad. Esto permitió identificar las áreas de oportunidad con respecto a la implementación de las tutorías. Se apoyaron en entrevistas semiestructuradas realizadas a docentes y tutores de las tres instituciones. Los resultados arrojaron la falta de coordinadores y administradores que apoyen en el ejercicio de la tutoría, así como la necesidad de herramientas tecnológicas que faciliten a los tutores llevar acabo la acción tutorial. Además, se identificó que cada una de las escuelas tiene necesidades particulares ante el desarrollo de las tutorías (González, 2017). 


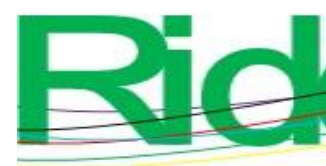

Revista Iberoamericana para la Investigación y el Desarrollo Educativo ISSN 2007 - 7467

\section{Metodología}

El tipo de estudio fue cualitativo, de alcance exploratorio y descriptivo (Hernández, Fernández y Baptista, 2014). Tuvo como objetivo identificar las necesidades en una escuela normal sobre la importancia de un programa de tutorías académicas, así como las posibilidades de su instrumentación desde la visión de sus actores: alumnos, docentes y directivos.

\section{Participantes}

Se contó con la participación de 47 alumnos, 20 profesores y los 3 directivos del plantel. El número de participantes obedeció a que fue una invitación voluntaria, tanto para los alumnos como para los profesores.

\section{Procedimiento y materiales}

El proceso de trabajo de campo se desarrolló en dos etapas para cada uno de los grupos de participantes. Primero, se realizaron tres talleres dirigidos a alumnos, docentes y directivos sobre el programa de tutorías académicas en las instituciones de educación superior. Esto, con la intención de contextualizar este tipo de estrategias dentro de la institución. Cada taller duró seis horas.

En una segunda etapa, se realizaron tres entrevistas grupales: una con los alumnos, otra con el personal docente y la última con los directivos. Estas se apoyaron en una guía de entrevista semiestructurada conformada por cuatro dimensiones: la importancia de la tutoría, antecedentes de la tutoría en la institución, tutoría en la actualidad dentro de la institución (no oficial) e instrumentación de la tutoría. La tabla 1 ilustra el proceso de trabajo de campo. 


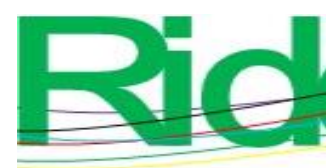

Revista Iberoamericana para la Investigación y el Desarrollo Educativo ISSN 2007 - 7467

Tabla 1. Proceso de trabajo de campo

\begin{tabular}{|c|c|l|c|l|l|}
\hline Etapa & Estrategia & \multicolumn{1}{|c|}{ Objetivo } & Cantidad & Participantes & \multicolumn{1}{|c|}{ Materiales } \\
\hline 1 & Taller & $\begin{array}{l}\text { Contextualizar la } \\
\text { tutoría académica } \\
\text { dentro de la } \\
\text { institución. }\end{array}$ & 3 & $\begin{array}{l}\text { Alumnos } \\
\text { Docentes } \\
\text { Directivos }\end{array}$ & $\begin{array}{l}\text { Presentación en } \\
\text { diapositivas y } \\
\text { formatos. }\end{array}$ \\
\hline 2 & $\begin{array}{c}\text { Entrevista } \\
\text { grupal }\end{array}$ & $\begin{array}{l}\text { Identificar la } \\
\text { importancia de la } \\
\text { tutoría, así como } \\
\text { los antecedentes, } \\
\text { acciones y posible } \\
\text { instrumentación en } \\
\text { la institución. }\end{array}$ & 3 & $\begin{array}{l}\text { Alumnos } \\
\text { Docentes } \\
\text { Directivos }\end{array}$ & $\begin{array}{l}\text { Guía de } \\
\text { entrevista } \\
\text { semiestructurada, } \\
\text { grabadora. }\end{array}$ \\
\hline
\end{tabular}

\section{Fuente: Elaboración propia}

Finalmente, se analizó la información obtenida en las entrevistas con el programa MaxQDA the arts of data analysis, en su versión 2018, para identificar la generación de las categorías ilustradas en la tabla 2.

\section{Resultados}

El análisis del contenido de las tres entrevistas grupales permitió generar categorías dentro de cada una de las dimensiones planteadas al inicio en la guía de entrevista. En la tabla 2, se muestran las dimensiones y sus respectivas categorías, las cuales se describen en este apartado de resultados, estructurado de acuerdo con las cuatro dimensiones. 


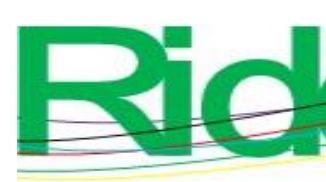

Revista Iberoamericana para la Investigación y el Desarrollo Educativo ISSN 2007 - 7467

Tabla 2. Esquema general de dimensiones y categorías

\begin{tabular}{|c|c|}
\hline Dimensiones & Categorías \\
\hline Importancia de la tutoría & $\begin{array}{ll}\text { - } & \text { Desarrollo del docente } \\
\text { - } & \text { Desarrollo del alumno } \\
\text { - } & \text { Guía para el alumno } \\
\text { - } & \text { Medidas precautorias } \\
\end{array}$ \\
\hline Antecedentes de la tutoría & $\begin{array}{l}\text { - Solicitudes para su consolidación } \\
\text { - Razones por las que no se } \\
\text { consolida } \\
\text { - Acciones realizadas }\end{array}$ \\
\hline Tutoría no oficial & $\begin{array}{ll}\text { - } & \text { Actores } \\
\text { - } & \text { Lugares } \\
\text { - } & \text { Modalidades } \\
\text { - } & \text { Funciones } \\
\text { - } & \text { Actividades } \\
\text { - } & \text { Limitaciones }\end{array}$ \\
\hline Instrumentación de la tutoría & $\begin{array}{l}\text { - } \text { Actores } \\
\text { - Perfil } \\
\text { - Funciones } \\
\text { - Estructura } \\
\text { - Condiciones de operación }\end{array}$ \\
\hline
\end{tabular}

Fuente: Elaboración propia

\section{Importancia de la tutoría}

En cuanto a la primera dimensión, importancia de la tutoría, los participantes manifiestan que esta es esencial en las IES, y señalan cuatro rubros que lo fundamentan (ver figura 1). El primero es la importancia que tiene la tutoría para el desarrollo de los docentes, ya que ellos pueden lograr un perfil idóneo como profesores de educación superior y de esta manera pueden participar en los cuerpos académicos, como lo expone el participante 1 en la entrevista con los profesores: "Es básico en nuestro perfil superior, ya que sin ella no puedes tener el perfil deseable un maestro de tiempo completo, para participar en los cuerpos académicos, es un requisito obligatorio, por eso es importante".

El segundo rubro es la importancia que tiene para el desarrollo del alumno en diferentes áreas como las académicas, tal como lo menciona el participante 9 de la entrevista de profesores:

Y cada uno de los planes de la licenciatura nos exigen que el alumno tiene que desarrollar un perfil de egreso y si no estamos cumpliendo con esos principios y con esos perfiles de egreso de nuestros alumnos, estamos de alguna manera 


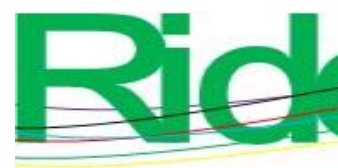

Revista Iberoamericana para la Investigación y el Desarrollo Educativo ISSN 2007-7467

no quedando bien con la sociedad a donde ellos van a ir a trabajar, sobre todo con los niños y jóvenes que van atender.

Asimismo, expresan la importancia de consolidar áreas de oportunidad que se identifican en los alumnos; en especial, el desarrollo integral de ellos.

En el tercer rubro, los participantes mencionan la importancia de guiar al alumno en su transcurso dentro de la institución, obteniendo una orientación y un apoyo en el acompañamiento, como hace mención el participante 2 de la entrevista de los alumnos de segundo semestre: “Además como es importante que vaya alguien contigo de la mano, porque sí, a veces te agobias por muchas cosas que tienes que hacer”.

Igualmente, mencionan que es importante porque se resuelven diferentes problemáticas o dudas sobre alguna situación y obtienen información por medio de sus tutores sobre diversas cuestiones que se manejan en la institución, como lo menciona el participante 2 de la entrevista de alumnos de segundo semestre: "Y si es una persona, un docente, es más fácil que ellos tengan información porque la mayoría ya pasó por aquí, como son docentes ya saben cómo se maneja la escuela, te pueden dar información más concretas, más adecuadas".

En el último aspecto, señalan la importancia que tiene la tutoría en cuestiones de medidas precautorias, puesto que se pueden evitar diferentes situaciones, como la deserción escolar, el rezago educativo, entre otras, que influyen en el desarrollo integral del estudiante. Al respecto, el participante 2 de la entrevista con directivos indica: "Tratar de prevenir que la situación llegue a ser problema, si son situaciones familiares, económica, ver la manera de cómo se puede acercar al alumno con la persona que tenga que hacerlo correspondiente". También mencionan la canalización con expertos que puedan atender la situación y el seguimiento que el tutor brinde al alumno durante toda su trayectoria.

Con base en lo anterior, alumnos, profesores y directivos consideran la importancia de la tutoría académica, puesto que lo mencionado por los participantes impacta en diversas cuestiones del desarrollo formativo de los alumnos dentro de la institución. 

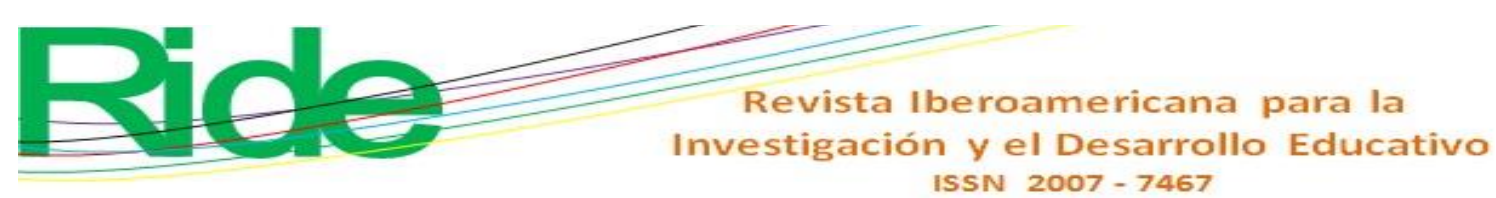

Figura 1. Importancia de la tutoría

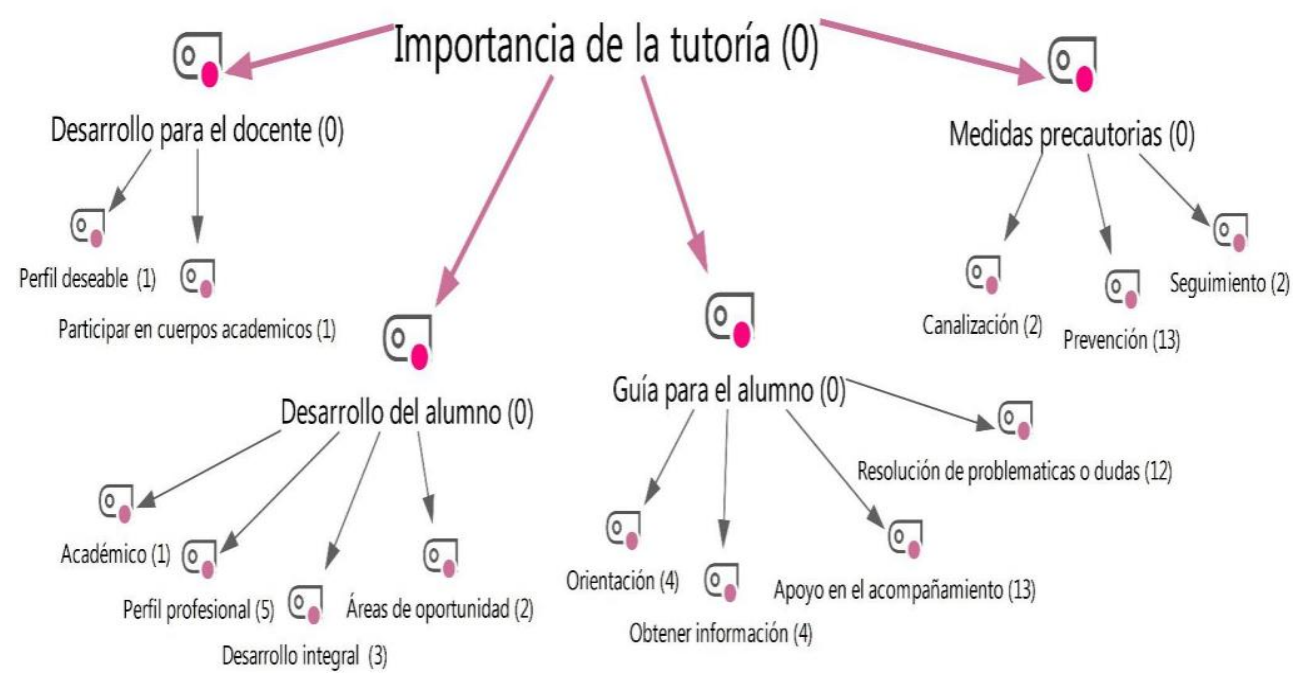

Fuente: Elaboración propia

\section{Antecedentes de la tutoría}

En la dimensión antecedentes de la tutoría, los participantes manifiestan tres rubros sobre acontecimientos que han ocurrido en relación con la tutoría académica dentro de la institución: acciones ya realizadas en torno a la tutoría, razones por las que no se consolida la tutoría y solicitudes planteadas para su consolidación (ver figura 2).

En cuanto al primer factor, los participantes indican que hace tiempo se implementó como una materia, por lo que se le fijó un horario. En este sentido, el participante 11 de la entrevista con alumnos de octavo semestre comenta: "Nos llegaron a dar; bueno, haz de cuenta como materia, pero era tutoría y era un maestro que nos estaba dando, pero realmente yo nunca miré que le estuvieran dando el enfoque o tuviera el verdadero fin de lo de la tutoría".

Coincidiendo con lo dicho por el participante anterior, el número 23 de la entrevista con profesores comenta: "Digamos, los alumnos que tenemos hoy en sexto semestre fueron los últimos que en segundo tuvieron tutorías y ellos ahora están en prácticas; sin embargo, ahí fue el último momento cuando se organizó, o sea, no hace cinco, hace dos años y algo". En otras palabras, se confirma el intento de realizar tutorías académicas, aunque al parecer fue implementada - tal vez por el hecho de ser una institución formadora de formadorescomo una materia de tutorías semejante a la educación básica. 


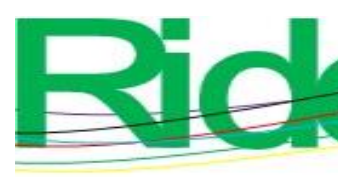

Revista Iberoamericana para la
Investigación y el Desarrollo Educativo
ISSN $2007-7467$

Además, refieren la existencia de un coordinador de tutoría, lo que coincide con las acciones sobre los intentos de consolidación de las tutorías académicas en la institución. Para esto, el participante 1 de la entrevista con directivos expresa: "Hubo un proyecto de instalar el proyecto de tutorías, estaban ahí contemplados algunos maestros aquí del área de psicopedagogía y sí llegaron a establecer algunas líneas”.

Aunado a esto, en los documentos proporcionados por la institución, se ve reflejado un diagnóstico realizado por la coordinadora de tutorías, el cual se basó en cuestionarios aplicados a alumnos y a profesores con un propósito: "Sentar las bases que puedan ir generando nuevos caminos y que cada uno podamos comprometernos con la parte que nos corresponde". Respondiendo al diagnóstico, la coordinación genera un proyecto de tutorías a manera de pilotaje, donde se marcan, de manera general, las acciones que se deben llevar a cabo.

Para el segundo rubro, se mencionan las razones por las que no se han consolidado las tutorías académicas dentro de la institución. Los participantes consideran que las causas se relacionan con la falta de un programa establecido con lineamentos para guiar el quehacer como tutores, escaso interés por parte de algunos profesores y directivos de la institución, así como cambios de directivos, los cuales no dan continuidad a los procesos empezados.

En lo referente a la falta de interés, el participante 1 de la entrevista de los directivos indica: "Pero por lo menos yo que fui tutor nunca ejercimos la tutoría y yo sé el caso de otros compañeros que fuimos elegidos tutores, no realizamos esa función como tal”. Por otra parte, el participante 17 de la entrevista de los profesores dice: "A veces porque los docentes no quieren participar por diferentes circunstancias". Esto reafirma lo mencionado en la categoría de falta de interés, puesto que los mismos participantes lo manifiestan.

Por último, en la categoría solicitudes para su consolidación, se establecen cuatro códigos: programa estatal (es decir, un programa de tutorías para las escuelas normales del estado), espacios (los actores indican que se han solicitado ambientes para llevar a cabo el servicio de la tutoría académica), capacitación y gestión de recursos. 


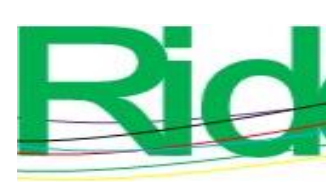

Revista Iberoamericana para la
Investigación y el Desarrollo Educativo ISSN $2007-7467$

Figura 2. Antecedentes de la tutoría

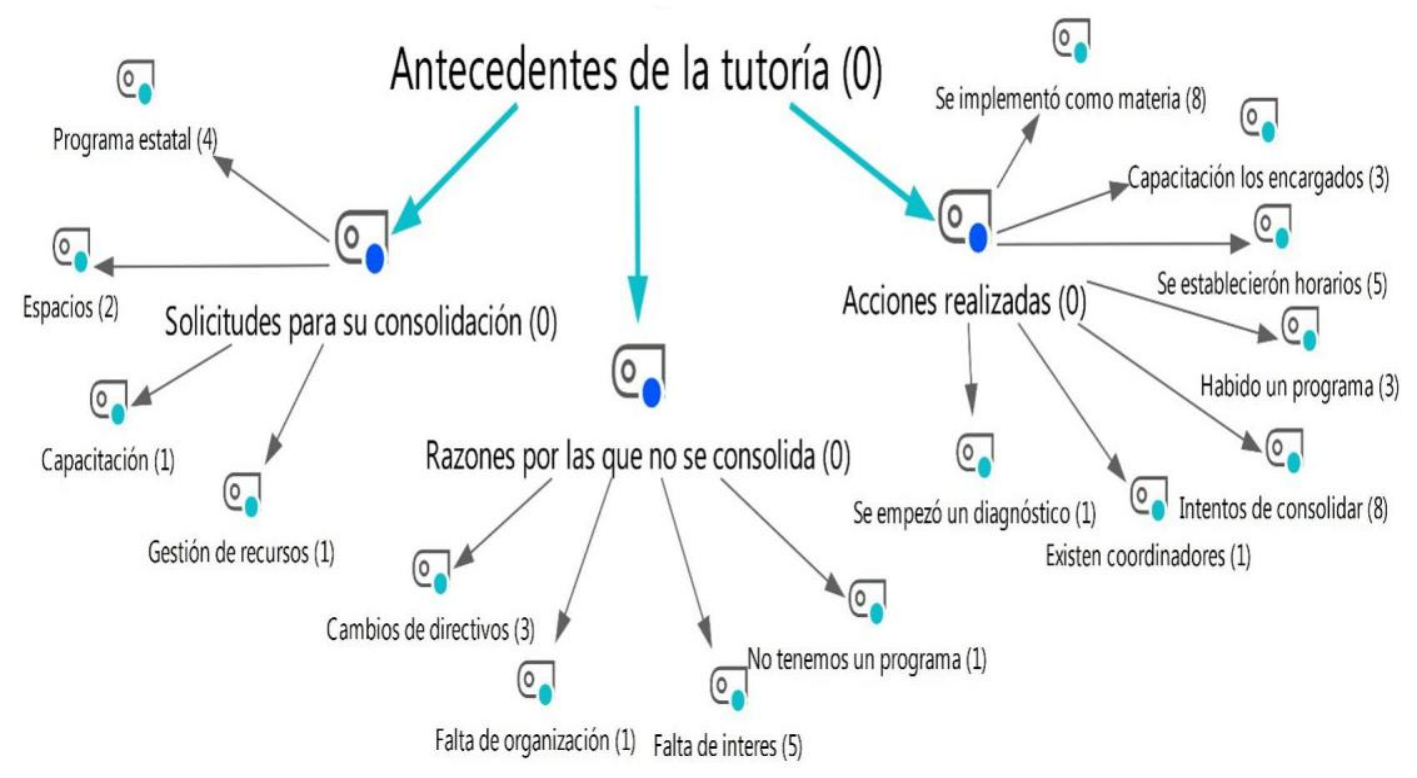

Fuente: Elaboración propia

\section{Tutoría no oficial}

En la dimensión tutoría no oficial surgieron seis categorías, vinculadas con las acciones llevadas a cabo en la institución, identificadas como labores de la tutoría académica (ver figura 3). De una de esas categorías (los actores) se desprenden siete códigos, que muestran los agentes que llevan a cabo la tutoría no oficial actualmente dentro de la institución, es decir, asesor de tesis, directivos, profesores de la práctica, maestros, prefectos, psicopedagogos y coordinadores. Los participantes indican que cada uno de estos apoya o acompaña durante el transcurso por la escuela.

La segunda categoría (lugares) se refiere a aquellos espacios donde se da la tutoría, por lo que nacen dos subcategorías: lugares formales e informales. Dentro de los formales se codifican el salón de clases y la oficina-cubículo. Los informales se vinculan con el estacionamiento, los pasillos, la cafetería, las áreas verdes y los lugares espontáneos. Sobre este último sitio, uno de los alumnos expresa: "Donde te los encuentres, los veas y los puedas abordar es donde te dan la orientación para ayudarte".

En lo correspondiente a la categoría modalidades, los participantes manifiestan cuatro códigos: tutoría grupal (en mayor medida), tutoría individual, tutoría presencial y tutoría virtual (en menor medida). Al respecto, se puede inferir que, debido a que son más las tutorías 


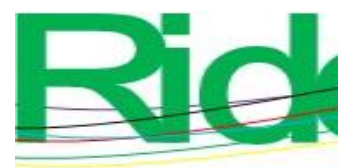

Revista Iberoamericana para la Investigación y el Desarrollo Educativo ISSN 2007 - 7467

grupales, los profesores suelen dar una orientación a los jóvenes cuando los atienden durante una clase, como indica el participante 27 de la entrevista de los profesores: "Normalmente en los grupos detectamos necesidades y los atendemos precisamente a veces dejando o complementando, aunque no vayan al contenido o al programa".

En la categoría funciones, se identifican ocho códigos, todos referentes a las acciones que realizan los docentes en cuestión de tutorías, es decir: canalización al área de psicopedagogía cuando se detectan algunos casos; apoyo ante diversas situaciones cuando así es requerido; los actores que realizan las acciones de tutorías brindan confianza; brindan una guía; asesorías académicas; ayudan a buscar soluciones a las problemáticas presentadas; detectan necesidades, y ayudan a trabajar áreas de oportunidad de los estudiantes.

La penúltima categoría se refiere a las actividades desarrolladas en torno a la tutoría, las cuales, algunas veces, deben ser ejecutadas dejando de lado el contenido de la materia. Al respecto, el participante 27 de la entrevista de los profesores indica: "Normalmente en los grupos detectamos necesidades y los atendemos precisamente, a veces dejando o complementando, aunque no vayan al contenido o al programa". Esto demuestra las acciones que los docentes realizan en cuestión de tutorías y las modalidades en que las realizan.

Por último, la categoría limitaciones se asocia con los obstáculos a los que se ha enfrentado la tutoría no oficial, la cual, por no estar oficializada, es interrumpida o se realiza de manera inadecuada y con información inconveniente. Por lo tanto, en esta dimensión se visualiza que, a pesar de que no existe un programa o una estructura formal, los docentes cumplen con dicho servicio, el cual es inherente a la vocación de ser maestro, como lo explica el participante 27 de la entrevista de profesores: "Yo considero también que es una función natural del maestro". 
Figura 3. Tutoría no oficial

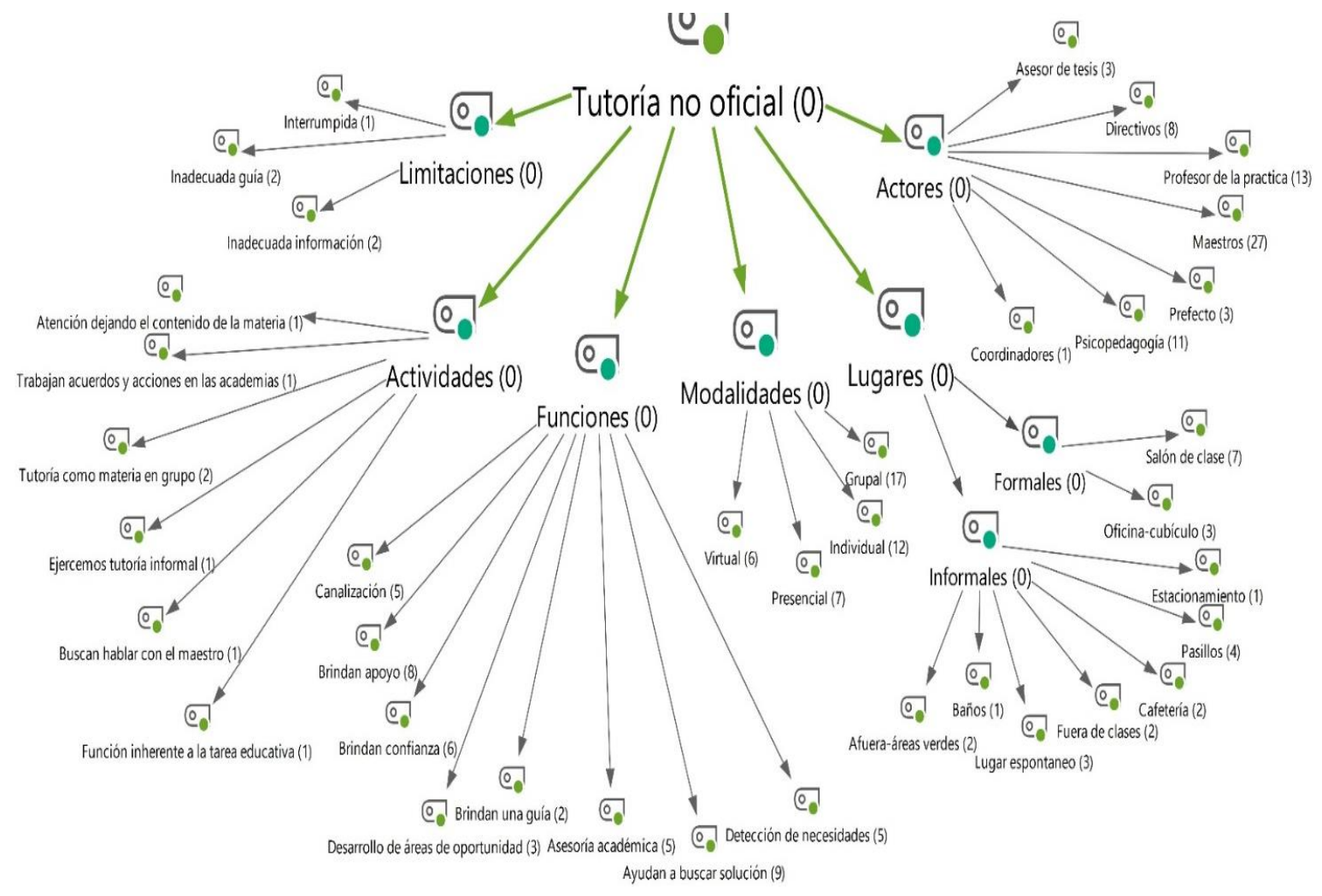

Fuente: Elaboración propia

\section{Instrumentación de la tutoría}

En la dimensión instrumentación de la tutoría se establecen cinco categorías: los actores que se consideran para el desarrollo de la tutoría ya establecida; los perfiles que deben de tener estos actores; las funciones que deben desarrollar; la estructura que debe tener la implementación del servicio de tutorías y, por último, todas las condiciones que se deben de tomar en cuenta para su operación (ver figura 4).

En lo correspondiente a actores, surgen cinco códigos: maestros nuevos (esto se refiere a que las funciones del tutor deben ser realizadas por docentes que no conozcan a los estudiantes $\mathrm{y}$, por tanto, que no tengan una predisposición negativa hacia ellos). Los siguientes códigos son maestros frente a grupo y maestros no frente a grupo (aquí hay una contradicción, puesto que hay participantes que mencionan que el tutor no debe estar frente a grupo para que atienda las tutorías, pero también mencionan que debe ser el maestro frente a grupo para que conozca a sus tutorados). De igual forma, se indicó que los maestros de práctica también pueden impartir la tutoría por su conocimiento sobre el campo práctico. 


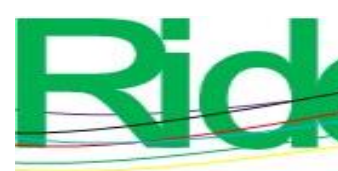

Revista Iberoamericana para la Investigación y el Desarrollo Educativo ISSN 2007 - 7467

Asimismo, se mencionó que los maestros que no son de base también pueden fungir como tutores, ya que conocen a los estudiantes, independientemente de que no tengan tanta información sobre la institución como los maestros de base o tiempo completo.

En la categoría perfil de los actores, se manifiestan códigos relacionados con la vocación por parte del profesor: voluntad (referente a que el tutor debe ser voluntario, y no impuesto); tutor responsable; empático; ético; comprometido; que tenga disponibilidad de atender a sus tutorados; que sea una persona que inspire confianza; que sea un tutor preparado, por medio de capacitaciones, y que tenga experiencia en el acompañamiento con los estudiantes.

Los participantes expresan que, para la operación de las tutorías, se necesitan diferentes aspectos, los cuales se codifican en los siguientes: compromiso de los directivos; partir de un diagnóstico; organización; trabajo colegiado (refiriéndose a trabajo en equipo por parte de los docentes y directivos de la institución); construcción de un programa; realizar planeación de la labor tutorial; oficializar la tutoría; que sea obligatoria; que se sensibilice a sus agentes sobre la importancia del servicio de la tutoría; capacitar y definir a los docentes que serán tutores; que exista una comunicación entre los agentes, y que se le dé continuidad al proceso de tutorías. Sobre lo anterior, el participante 45 de la entrevista de alumnos de octavo semestre expresa:

Si no que la tutoría esta, es como especializada para cada alumno, para los diferentes problemas que se dan en su contexto. Entonces que realmente se implemente aquí en la institución una instancia o un lugar con tutores, con tutores específicos, posiblemente sean maestros frente a grupo, pero que tengan la función de tutor, no de tutor en un semestre, tutor en el otro, tutor aquí, tutor allá, sino que realmente este espacio de tutoría, esos cubículos de tutoría, esos lugares de tutoría, esa información o apoyo de tutoría.

Con respecto a las funciones de los tutores, hubo coincidencia al indicar que los tutores deben realizar un conjunto de actividades propias de la tutoría, desde el enfoque de acompañamiento de estudiantes y no confundirlas con otro tipo de actividades. Se debe conocer a los estudiantes, es decir, realizar su labor conforme a un perfil particular; también mencionaron la importancia de que guíe y apoye durante el proceso formativo, ya sea desde el plano de ofrecer información de determinados procesos y actividades, hasta la orientación 

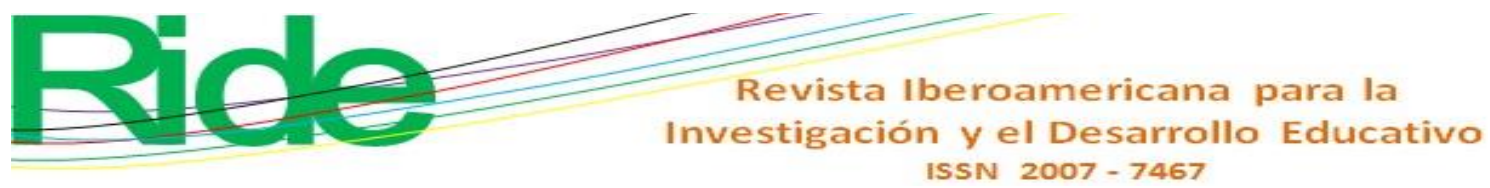

para la solución de problemáticas que se presenten durante su proceso formativo, también se manifiestan por un tutor que les oriente a la formación integral y que se conduzca con valores.

Sobre la estructura para la formalidad de un programa de tutorías, los participantes primero distinguen la importancia de que la función se formalice en los horarios de los tutores, es decir que forme parte de una actividad reconocida en sus diversas funciones, así mismo que existan espacios físicos (cubículos, por ejemplo) para la atención de estudiantes y finalmente que se definan las la modalidades o tipologías en la que se desarrollará el programa: presencial, individual y grupal.

En síntesis, se puede asegurar que los diferentes agentes involucrados en las entrevistas tienen interés por el desarrollo de las tutorías académicas dentro de la institución, pero enfatizando que sea un programa propio, basado en sus necesidades.

Figura 4. Instrumentación de la tutoría

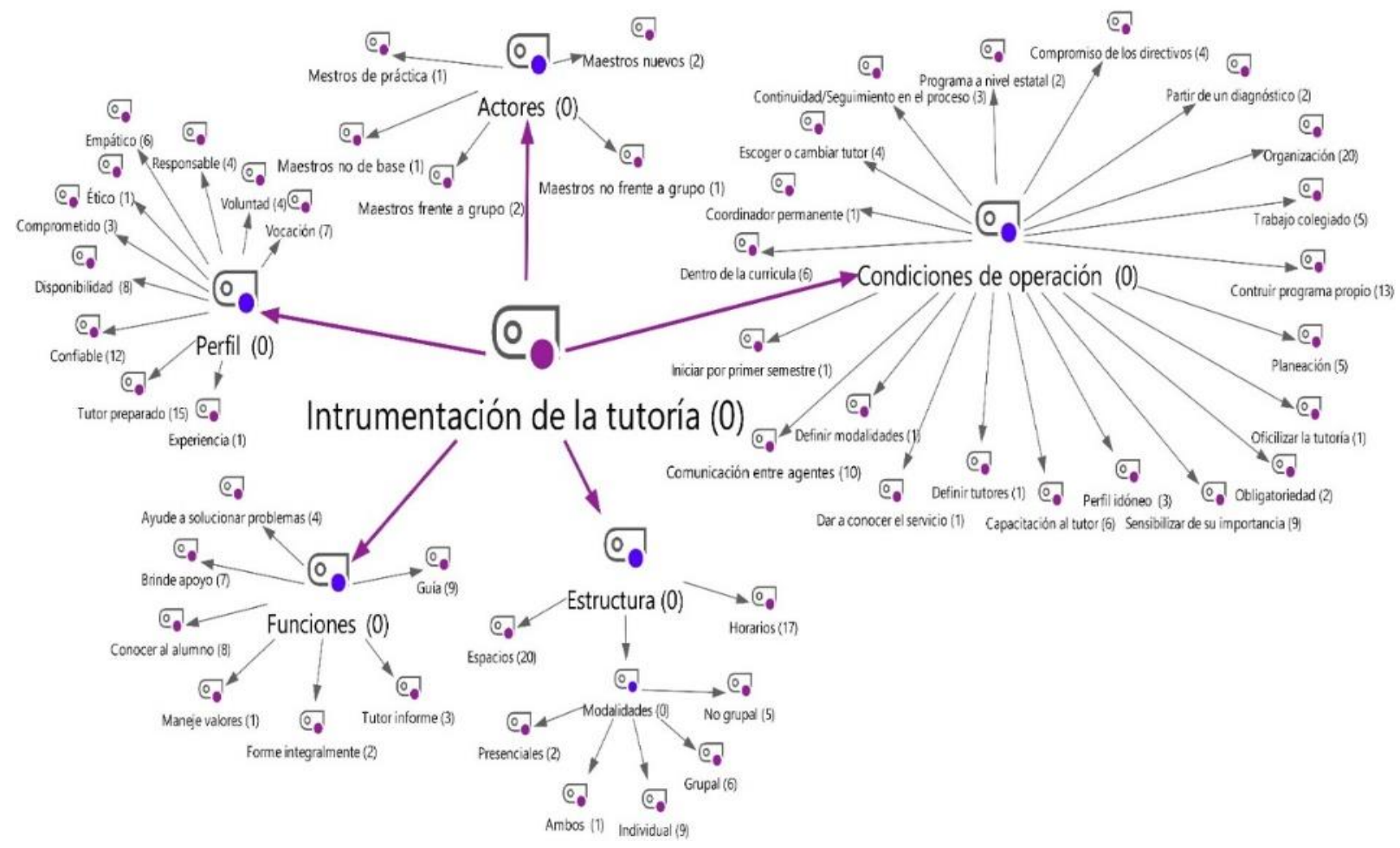

Fuente: Elaboración propia 


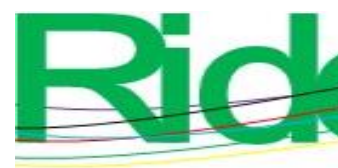
Revista Iberoamericana para la
Investigación y el Desarrollo Educativo
ISSN $2007-7467$

\section{Discusión}

El presente estudio se caracterizó por tres aspectos relevantes: reunir la opinión de docentes, alumnos y directivos sobre la importancia de las tutorías académicas para la institución, encontrar las coincidencias de las opiniones de estos tres grupos y aportar a la delimitación colegiada sobre la posible instrumentación de este tipo de estrategias.

En tal sentido, se destaca el cumplimiento del objetivo del estudio, que buscó el desarrollo de un diagnóstico para orientar la elaboración de un programa de tutorías, trabajo que se caracterizó por la colegiación de los distintos actores involucrados. Al respecto, es importante destacar tres hallazgos significativos sobre las tutorías en la institución seleccionada: el primero consiste en un consenso generalizado sobre la importancia de las tutorías y la necesidad de diseñar un programa formal; el segundo tiene que ver con los antecedentes de este tipo de programas en la institución, el cual, por diferentes razones, no se desarrolló; y el tercero revela que dicho servicio de tutorías se está ejecutando, aunque de manera informal, es decir, sin lineamientos establecidos y sin contar con espacios o personal exclusivo para tal fin.

Por ello, y al igual que en otras escuelas normales, es evidente la necesidad de contar con un servicio de tutoría formalizado que permita promover su instrumentación, lo que disminuiría su falta de claridad (Ponce, Aceves y Boroel, 2020). En efecto, contar con condiciones institucionales adecuadas para instrumentar la tutoría permitirá el desarrollo de la estrategia y, paulatinamente, su mejora (Romo, 2014).

Se espera que este trabajo sea una aportación al campo de las tutorías académicas en el contexto de escuelas normales, tanto en la clarificación del desarrollo de este tipo de estrategias como en el aporte metodológico para la investigación y el desarrollo de diagnósticos para emprender este tipo de procesos.

Finalmente, la limitación principal del estudio radicó en su carácter particular para una escuela normal, por lo que se invita al uso de estos resultados como antecedente para emprender proyectos más amplios e integrales. 


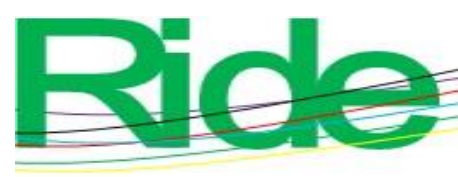

Revista Iberoamericana para la Investigación y el Desarrollo Educativo ISSN $2007-7467$

\section{Conclusiones}

La tutoría ha fomentado diversos desarrollos y avances en las IES donde se ha instrumentado; en particular, aquellas que, desde el nacimiento de la propuesta de ANUIES, han seguido su progreso. Sin embargo, en el caso de las escuelas normales, aún no se cuenta con evidencia amplia que documente el desarrollo y sus resultados. No obstante, existen algunos estudios que demuestran que dicha estrategia ha favorecido, especialmente, la formación de los estudiantes, aun cuando su implementación ha sido heterogénea, lenta y con diversas dificultades.

Por eso, las conclusiones principales de este trabajo se orientan a la importancia de que las escuelas normales cuenten con un programa de tutorías. De acuerdo con los resultados obtenidos, su importancia radica en cuatro aspectos esenciales: "desarrollo del alumno" y "guía para el alumno". Estos dos primeros se refieren a los beneficios que subyacen en el apoyo brindado a los alumnos para su proceso académico y para su desarrollo integral. El tercer aspecto sería el "desarrollo de los docentes", pues la tutoría se puede convertir en un estímulo para procurar alcanzar el perfil deseable. Por último, el elemento "medidas precautorias" hace énfasis en que a través de la tutoría se puede prevenir, canalizar y/o abordar problemáticas que pudieran afectar u obstaculizar el proceso formativo del estudiante.

En este sentido, se considera fundamental que para la elaboración de un programa de esta naturaleza se cuente con la participación de los distintos actores-usuarios de este tipo de estrategias, así como valorar el contexto de aplicación a partir de las posibilidades y realidades de cada plantel, por supuesto respetando lo establecido en los planes y programas de estudios de las escuelas normales.

En relación con la segunda interrogante planteada al inicio del estudio, se llegó a la conclusión de que sí hay antecedentes de un programa de tutorías, el cual existió hace años, aunque no se implementó. Por otro lado, se concluye que actualmente sí se ofrece el servicio de tutorías por parte de los docentes a los alumnos, aunque de manera informal, ya que no se cuenta con lineamientos que especifiquen las condiciones de su operatividad. Esto, por ende, representa una futura línea de trabajo.

Respondiendo a la tercera interrogante que guio el presente estudio, se concluye que la implementación de un programa de tutorías, desde la visión de los usuarios, exige aspectos como compromiso por parte de los directivos, trabajo colegiado, oficializar la tutoría 


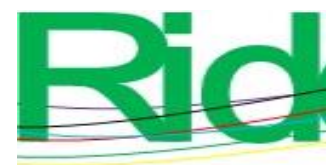

Revista Iberoamericana para la
Investigación y el Desarrollo Educativo
ISSN $2007-7467$

(definiendo modalidades, espacios y funciones del tutor), sensibilidad y capacitación de los tutores, así como seguimiento al proceso de tutorías. Estos aspectos darán sustento al programa y permitirán su implementación y continuidad.

Finalmente, en cuanto al perfil idóneo para ser tutor de la escuela normal seleccionada en este trabajo, los participantes consideran que el docente se debe caracterizar por su vocación, responsabilidad, empatía, compromiso, formación e interés para realizar dicha función de forma voluntaria. Todas estas conclusiones ofrecidas permiten sustentar y guiar la creación del programa de tutorías académicas para la escuela normal dada su trascendencia para la formación de los estudiantes.

\section{Futuras líneas de investigación}

En principio, se debe trabajar en la evaluación del impacto de los programas de tutoría en las IES (en general), así como en las escuelas normales, en particular, ya que son escasos los estudios en dicha área. Asimismo, se debe repensar la forma de instrumentar las tutorías en las distintas instituciones, las cuales deben tomar en cuenta los cambios de escenarios formativos. Asimismo, es importante explorar la incorporación de este tipo de estrategias en la diversidad de IES, en especial en aquellas donde exista limitada evidencia sobre su desarrollo. 


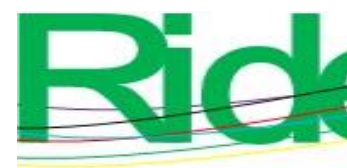
Revista Iberoamericana para la
Investigación y el Desarrollo Educativo
ISSN $2007-7467$

\section{Referencias}

Ahumada, F. y Obregón, C. (2015). La tutoría profesional: una estrategía para favoreser la formación de docentes a partir del desarrollo de la autoestima. Ra Ximhai, 11(4), 7590. Recuperado de http://www.redalyc.org/pdf/461/46142596004.pdf

Castillo, y Cabrerizo, J. (2011). Evaluación de la intervención socioeducativa. Agentes, ámbitos y proyectos. Madrid: Pearson.

Diario Oficial de la Federación (2018). Acuerdo 14/07/18. Estados Unidos Mexicanos, 03 de agosto de 2018. Recuperado de https://www.dof.gob.mx/nota_detalle.php?codigo=5533902\&fecha=03/08/2018

Dirección General de Educación Superior Universitaria [DGESU] (s. f.). Programa para el desarrollo profesional docente, para el tipo superior. México: DGESU. Recuperado de http://www.dgesu.ses.sep.gob.mx/PRODEP.htm

Fresán, M. y Romo, A. (2011). Programas institucionales de tutoría: una propuesta de la Anuies. México: Anuies.

García, B., Ponce, S., García, M., Caso, J., Morales, C., Martínez, S., Serna, A. y Aceves, Y. (2016). Las competencias del tutor universitario: una aproximación a su definición desde la perspectiva teórica y de la experiencia de sus actores. Perfiles Educativos, 38(151), 104-122. Recuperado de https://www.redalyc.org/pdf/132/13243471007.pdf

García, F., Trejo, M., Flores, L. y Rabadán, R. (2010). Una estrategia educativa que potencia la formación de profesionales. México: LIMUSA.

Gobierno de España (1989). Libro blanco para la reforma del sistema educativo. España: Ministerio de Educación y Ciencia. Recuperado de https://sede.educacion.gob.es/publiventa/libro-blanco-para-la-reforma-del-sistemaeducativo/administracion-educativa/913

González, V. (2017). Práctica de las tutorías en las escuelas normales de Mérida. Memoria del Congreso Nacional de Investigación sobre Educación Normal (1). Recuperado de http://www.conisen.mx/memorias/memorias/4/C180117-H146.docx.pdf

Hernández, R., Fernández, C. y Baptista, P. (2014). Metodología de la investigación. México: McGRAW-HILL.

Mapén, T., Pérez, M. y López, A. (2015). Evaluación del programa de tutoría en la Escuela Normal de Educación Preescolar "Rosario María Gutiérrez Eskildsen". Perspectivas 


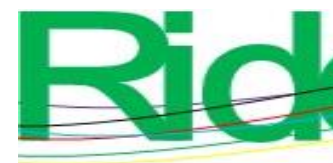

Docentes,

(59),

13-18.

Recuperado

de http://revistas.ujat.mx/index.php/perspectivas/article/view/1304

Martínez, R., Cortés, A. y Contreras, E. (2017). La tutoría en la práctica. Propuestas de mejora. Memoria del Congreso Nacional de Investigación sobre Educación Normal (1). Recuperado de http://www.conisen.mx/memorias/memorias/4/C180117H101.docx.pdf

Mino, N., Castellanos, A, y Gómez, C. (2016). El programa de tutoría en la Escuela Normal Rural: realidades de su implementación. Memoria del Séptimo Encuentro Nacional de Tutoría. Recuperado de https://oa.ugto.mx/wp-content/uploads/2016/11/oa-rg0000513.pdf

Pantoja, A. (2013). La acción tutorial en la escuela. Madrid: Síntesis.

Ponce, S., Aceves, Y. y Boroel, B. (2020). Exploración del comportamiento de un instrumento para evaluar la función de tutoría académica en el contexto de escuelas normales. Revista Iberoamericana de Evaluación Educativa, 13(1), 139-155. Doi: http://dx.doi.org/10.15366/riee2020.13.1.006

Quintanal, J. y García, M. (2012). La tutoría en la escuela. Madrid: CCS.

Ramírez, R., Campos, R. y Rodríguez, R. (2013). El estudiante normalista en el programa de tutoría. Revista Iberoamericana para la Investigación y el Desarrollo Educativo, (10). Recuperado de http://ride.org.mx/111/index.php/RIDESECUNDARIO/issue/view/3

Romo, A. (coord.) (2014). Los programas institucionales de tutoría: actores, procesos y contextos. México: ANUIES.

Sariñana, S. y Sariñana, V. (2016). Desarrollo de habilidades y actitudes mediante la tutoría subgrupal. Memoria del Séptimo Encuntro Nacional de Tutoría. Recuperado de https://oa.ugto.mx/desarrollo-de-estructuras-matematicas-repetando-nivelescognitivos.html

Serna, A. (2010). La tutoría académica desde la mirada de los alumnos. México: Universidad Autónoma de Baja California.

Soto, Y., Tequida, M. y Chávez J. (2017). Función de totoría: una mirada desde la práctica profesional. Memoria del Congreso Nacional de Investigación sobre Educación Normal (1). Recuperado de http://www.conisen.mx/memorias/memorias/2/C210117-J154.docx.pdf 
Tejada, M. (2016). La tutoría académica en el proceso de formación docente. Opción, 32(13), 879-899. Recuperado de http://www.redalyc.org/pdf/310/31048483042.pdf

\begin{tabular}{|l|l|}
\hline Rol de Contribución & Autor (es) \\
\hline Conceptualización & Salvador Ponce Ceballos \\
\hline Metodología & Astrid Olimpia Martínez Hernández \\
\hline Software & Astrid Olimpia Martínez Hernández \\
\hline Validación & Yaralín Aceves Villanueva \\
\hline Análisis Formal & Yaralín Aceves Villanueva \\
\hline Investigación & Astrid Olimpia Martínez Hernández \\
\hline Recursos & NO APLICA \\
\hline Curación de datos & Astrid Olimpia Martínez Hernández \\
\hline $\begin{array}{l}\text { Escritura - Preparación del } \\
\text { borrador original }\end{array}$ & Astrid Olimpia Martínez Hernández \\
\hline $\begin{array}{l}\text { Escritura - Revisión y } \\
\text { edición }\end{array}$ & Salvador Ponce Ceballos \\
\hline Visualización & NO APLICA \\
\hline Supervisión & Salvador Ponce Ceballos \\
\hline Administración de Proyectos & NO APLICA \\
\hline Adquisición de fondos & NLICA \\
\hline
\end{tabular}

